\title{
Minimal Deviation Melanoma
}

National Cancer Institute

\section{Source}

National Cancer Institute. Minimal Deviation Melanoma. NCI Thesaurus. Code C48612.

A melanocytic neoplasm displaying morphologic features that are intermediate between those of benign nevus and melanoma. It is characterized by a nodular architectural growth resembling a melanoma, and a loss of nevus cell maturation. The melanocytic cells forming the nodular growth are uniform, and they may display a high mitotic rate, but they do not show cytologic atypia. 